\title{
Comparison of Contact Lens and Intraocular Lens Correction of Monocular Aphakia During Infancy
}

\author{
Natalie Weil, MD ${ }^{1}$ and Scott R Lambert, MD² \\ 1. Pediatric Ophthalmology Fellow; 2. R Howard Dobbs Professor of Ophthalmology and Pediatrics, Emory University, Atlanta, Georgia, US
}

\begin{abstract}
The Infant Aphakia Treatment Study recommended primary intraocular lens (IOL) implantation be reserved for infants with parents unable to manage contact lenses. The best baseline predictor of a good visual outcome was private health insurance. IOL implantation was associated with more adverse events, but lower direct costs to parents.
\end{abstract}

\section{Keywords}

Infant Aphakia Treatment Study, primary intraocular lens, total direct costs, congenital cataracts, contact lens, iris prolapse, pupillary membranes

Disclosure: Natalie Weil, MD, and Scott R Lambert, MD, have no conflicts of interest to declare.

Open Access: This article is published under the Creative Commons Attribution Noncommercial License, which permits any noncommercial use, distribution, adaptation, and reproduction provided the original author(s) and source are given appropriate credit.

Received: January 10, 2015 Accepted: January 13, 2015 Citation: US Ophthalmic Review, 2015;8(1):21-2 DOl: 10.17925/usor.2015.8.1.21

Correspondence: Scott R Lambert, MD, R Howard Dobbs Professor of Ophthalmology and Pediatrics, Emory University, Atlanta, GA, US. E: scott.lambert@emory.edu

Support: Supported by National Institutes of Health (NIH) Grants U10 EY13272 and U10 EY013287 and in part by NIH Departmental Core Grant EY006360 and Research to Prevent

Blindness, Inc., New York, New York.

The Infant Aphakia Treatment Study (IATS) was a randomized, multicenter clinical trial that compared visual outcomes at age 4.5 years with or without intraocular lens (IOL) implantation in 114 children who underwent unilateral cataract surgery at 1 to $<7$ months of age. The study concluded that the visual outcomes were similar in both treatment groups, but there were more intraoperative and postoperative adverse events in the IOL group and, as a result, IOL implantation was only recommended if it was deemed likely that the parents of a child would not be able to manage contact lenses (CTLS). The most common adverse events in the IOL group were intraoperative iris prolapse (11\%), lens reproliferation into the visual axis (40\%), pupillary membrane (28\%), and postoperative correctopia (28\%). Nearly five times more patients in the IOL group required an additional intraocular surgery to clear the visual axis in comparison to the CTL group (68 \% versus $14 \%$ ). ${ }^{1,2}$ The majority of these adverse events occurred during the first year after cataract surgery. ${ }^{3}$ We also found the prevalence of glaucoma, strabismus, and stereopsis to be similar in the two treatment groups at age 5 years., ${ }^{2,4}$

While 1 year after cataract surgery, the total direct costs were $37 \%$ higher in the IOL group, by age 5 the total direct costs were nearly the same for both treatment groups. The higher costs in the IOL group at 1 year after cataract surgery were primarily due to the increased costs of additional intraocular surgeries. However, after the first postoperative year the costs were higher in the CTL group so that by age 5, the costs in the IOL group were only $7 \%$ higher than the CTL group. Most of the increased costs after the first postoperative year in the CTL group were due to the replacement of CTLS. Both groups of patients wore bifocal spectacles at age 2 and onward. The total cost of supplies (spectacles, CTLS, and occlusive patches) were nearly twice as high in the CTL group $(\$ 7,728)$ compared with the IOL group $(\$ 3,204)$. So while the total direct costs were still slightly higher at age 5 in the IOL group, the supply costs, which in most cases are borne by parents since most medical insurance policies do not cover these costs, were more than $\$ 4,000$ higher in the CTL group. ${ }^{5,6}$

We also analyzed whether there were baseline factors that could predict visual outcome. We found the age at which cataract surgery was performed to only be weakly associated with the age 4.5 visual outcome. Overall, while patients $<3$ months of age at the time of cataract surgery had an improved median visual acuity, the strongest predictor of visual outcome was whether the patient had private insurance. ${ }^{7}$ Patients covered by private insurance had a significantly better visual outcome compared with children covered by Medicaid.

Some have questioned whether there would have been fewer postoperative adverse events in the IOL group if steroids had been prescribed to be administered more frequently. It is unlikely that the more frequent instillation of topical corticosteroids would have affected the incidence of lens reproliferation into the visual axis. While it is possible that the more frequent administration of corticosteroids would have reduced the incidence of certain inflammatory complications such as pupillary membranes, we were unable to find an association 
between the frequency topical corticosteroids were prescribed and the prevalence of inflammatory membranes. ${ }^{8}$ It is possible that the incidence of adverse events would have been lower if more experienced pediatric cataract surgeons had performed the surgeries. However, it should be emphasized that all of the investigators participating in the Infant Aphakia Treatment Study were experienced pediatric cataract surgeons who were selected to participate in the clinical trial because of the high volume of pediatric cataract surgery at their clinical site.
The decision to implant an IOL in an infant $<7$ months of age with a visually significant unilateral congenital cataract is complicated. If parents have the financial resources to purchase CTLs and it is deemed that they can likely manage them, the IATS supports leaving these patients aphakic and correcting them optically with a CTL. However, there is still a subset of these patients who would likely benefit from IOL implantation. This decision must be carefully considered and made through an established patient-doctor relationship on an individual basis.
1. Infant Aphakia Treatment Study Group. A randomized clinical trial comparing contact lens and intraocular lens correction of monocular aphakia during infancy: Grating acuity and adverse events at age 1 year, Arch Ophthalmol, 2010:128:810-8.

2. Infant Aphakia Treatment Study Group. A randomized clinical trial comparing contact lens to intraocular lens correction of monocular aphakia during infancy: HOTV optotype acuity at age 4.5 years and clinical findings at age 5 years, JAMA Ophthalmo 2014;132:676-82.

3. Plager DA, Lynn MJ, Buckley EG, et al., Complications in the first 5 years following cataract surgery in infants with and without
IOL in the IATS, Am J Ophthalmol, 2014;158:892-8.

4. Hartmann EE, Stout AU, Lynn MJ, et al.; Infant Aphakia Treatment Study Group, Stereopsis results at 4.5 years of age in the Infant Aphakia Treatment Study, Am J Ophthalmol, 2015;159:64-70.

5. Carrigan AK, DuBois LG, Becker ER, Lambert SR, cost of intraocular lens vs contact lens treatment after unilateral congenital cataract surgery: Retrospective analysis at age 1 year, Ophthalmology, 2013;120:14-9.

6. Kruger S, DuBois L, Becker ER, et al. Infant Aphakia Treatment Study, Cost of intraocular lens vs. contact lens treatment after unilateral congenital cataract surgery in the Infant Aphakia Treatment Study: Retrospective analysis at age 5 years, Ophthalmology, 2015;122:288-92.

7. Hartmann EE, Lynn MJ, Lambert S, Baseline characteristics of the Infant Aphakia Treatment Study population: Predicting recognition acuity at 4.5 years of age, Invest Ophthalmol Vis SCi, 2015;56:388-95

8. Lambert SR, Plager DA, Buckley EG, et al.; Infant Aphakia Treatment Study, The Infant Aphakia Treatment Study: Was the high rate of intraoperative and postoperative adverse events in the intraocular lens group preventable? J AAPOS, in Press. 